\title{
Sensory Loss by Selected Whisker Removal Produces Immediate Disinhibition in the Somatosensory Cortex of Behaving Rats
}

\author{
M. Kathleen Kelly, ${ }^{1}$ George E. Carvell, ${ }^{1}$ Judith M. Kodger, ${ }^{2}$ and Daniel J. Simons ${ }^{2}$ \\ ${ }^{1}$ Department of Physical Therapy, School of Health and Rehabilitation Sciences, and '2Department of Neurobiology, \\ School of Medicine, University of Pittsburgh, Pittsburgh, Pennsylvania 15260
}

This study used extracellular unit recordings in behaving animals to evaluate thalamocortical response transformations in the rat whisker-barrel system. Based on previous acute studies using controlled whisker stimulation, we hypothesized that in a cortical barrel adjacent (non-principal) whiskers exert a net inhibitory effect. In contrast, in thalamic barreloid neurons, the effects of neighboring whiskers should be net facilitatory. We evaluated these hypotheses by recording unit activity at 21 sites in17 animals trained to explore a wire mesh screen with their whiskers. In the middle of the recording session, selected vibrissae were clipped close to the skin surface. The absence of whiskers surrounding the principal whisker was associated with a mean $20 \%$ increase in cortical activity and, conversely, a $37 \%$ decrease in the thalamic activity. Removal of the principal whisker resulted in a $50 \%$ decrease in cortical unit firing. Findings are consistent with the idea that, in the behaving animal, each barrel uses multi-whisker thalamic inputs and local inhibitory circuitry to sharpen the receptive field properties of its constituent neurons. Cortical disinhibition as a consequence of selective whisker removal is likely to be an important factor underlying altered receptive field properties in sensorydeprived animals.

Key words: barrel; whiskers; tactile behavior; EMG; inhibition; sensory deprivation; plasticity; active touch
The rodent somatosensory system provides a number of advantages for addressing issues related to the development and maintenance of normal sensory function. Simple whisker trimming in neonatal animals leads to permanent abnormalities in the cortical anatomy (Micheva and Beaulieu, 1995; Keller and Carlson, 1999) (but see Akhtar and Land, 1991), cortical receptive field properties (Simons and Land, 1987; Fox, 1992), and whisker-based discriminative behavior (Carvell and Simons, 1996). In adult animals, alterations in cortical receptive fields can be produced after a few days of whisker removal (Diamond et al., 1993). Elucidating the mechanisms responsible for these and other related phenomena (Buonomano and Merzenich, 1998) requires an understanding of local circuitry at different levels of the somatic afferent system.

Previously, we described differences in receptive field properties of thalamic "barreloid" and cortical "barrel" neurons (Simons and Carvell, 1989). The former had relatively large, excitatory multi-whisker receptive fields (Nicolelis et al., 1993) with weak inhibitory surrounds. In contrast, presumed excitatory neurons within barrels displayed smaller excitatory receptive fields and relatively strong inhibitory effects of adjacent whiskers (Brumberg et al., 1996). These results suggest that whisker removal should disinhibit neurons in neighboring cortical barrels but result in no net effect, or even a reduction in activity, in the

Received April 29, 1999; revised July 22, 1999; accepted Aug. 3, 1999.

This work was supported by National Science Foundation Grant IBN-9209490, National Institutes of Health Grant NS-19950, and a Viva J. Erickson scholarship and a Patricia Leahy memorial scholarship to M.K.K. from the American Physical Therapy Association. We thank Thomas Prigg for expert technical assistance, William W. Simons for developing the spike sorting software, and Peter Land and Harold Kyriazi for comments.

Correspondence should be addressed to M. Kathleen Kelly, Department of Physical Therapy, 6035 Forbes Tower, University of Pittsburgh, Pittsburgh, PA 15260.

Copyright @ 1999 Society for Neuroscience 0270-6474/99/199117-09\$05.00/0 corresponding thalamic barreloids. This idea is consistent with findings in 2-deoxyglucose (2DG) studies in freely exploring mice (McCasland and Woolsey, 1988). Metabolic labeling was greater in the $\mathrm{C} 3$ cortical column of mice acutely deprived of all but the principal whisker (C3) compared with levels in the C3 column of non-trimmed animals.

We interpret the above to mean that, within a barrel, the net effect of adjacent (non-principal) whiskers is normally inhibitory in behaving animals. This conclusion is tempered, however, by two lines of evidence. First, some single-unit studies have shown that, unlike effects we observed in fentanyl-sedated animals, adjacent whisker excitatory responses in the barrels of urethaneanesthetized rats are robust and of long duration (ArmstrongJames and Fox, 1987; Armstrong-James et al., 1992). Hence, between-whisker interactions within a barrel might be facilitatory. Second, in the barrel cortex of behaving hamsters, GABAergic neurons are more heavily 2DG-labeled than presumed excitatory cells (McCasland and Hibbard, 1997). Elevated metabolic activity in the C3 column of C3-only mice could therefore reflect increased cortical inhibition after adjacent whisker removal and not an increase in the activity of excitatory barrel neurons.

Here, we used neurophysiological approaches in behaving rats to test two hypotheses. If the net effect of adjacent whiskers is inhibitory, their removal should result in increased spike activity. Conversely, if the net effect of adjacent whiskers is excitatory, their removal should result in decreased activity. We assessed these alternatives by recording unit activity in animals trained to whisk a wire mesh screen. In the middle of the recording session, selected whiskers were clipped short. The first hypothesis was supported by the cortical unit data, whereas the second was supported by thalamic data. These results are important for understanding operations of local circuits in normal and in sensory-deprived animals. 


\section{MATERIALS AND METHODS}

\section{Animal training}

The purpose of the behavioral task was to promote extended periods of whisker system activation during a natural behavior. Adult female Sprague Dawley rats were trained to whisk and explore actively a wire mesh screen to search for a metal-tipped wand (3.5 $\mathrm{mm}$ in diameter) that protruded $1-2 \mathrm{~cm}$ through the mesh. Animals were rewarded with a small amount of powdered rat chow mixed with water and peanut butter.

Details of apparatus. The rat stood on an elevated platform and stretched across a gap of $7.5 \mathrm{~cm}$ to palpate the screen. The platform was $18.5 \mathrm{~cm}$ high, $24 \mathrm{~cm}$ long, and $18 \mathrm{~cm}$ wide at the back end, narrowing to $8 \mathrm{~cm}$ at the front end. The screen, which was supported in a wooden frame, consisted of wire mesh (grid size $6 \times 6 \mathrm{~mm}$ ) measuring $25.5 \mathrm{~cm}$ in width and $10.5 \mathrm{~cm}$ in height. A horizontal bar placed $8-9 \mathrm{~cm}$ above the platform and in front of the screen provided a means for the animal to stabilize its forepaws so that it could freely use its face to explore the full extent of the screen, combining whisking with lateral and vertical head movements. A Panasonic AG-530 video camera was positioned $30 \mathrm{~cm}$ above the screen to obtain an overhead view of the animal while it explored the screen. The shutter speed was set at $1 / 1000 \mathrm{sec}$. A time-date generator marked each video frame with the elapsed time of the experiment.

Initial training consisted of instituting the food deprivation schedule and acclimating the rat to handling. Over a period of a week, the animal was familiarized with the training apparatus by affording it access to food mash ad libitum while on the raised platform. During the second phase of training, the animal was introduced to the task itself. Trial onset was signified by a white noise audio signal. At first, the animal was encouraged to explore the screen by placing a small quantity of food mash on the wand. Contact of the animal's mouth with the wand completed an electrical circuit, terminating the white noise and presenting a tone that signaled availability of a food reward at the side of the platform. Eventually there was no need to place food on the wand because the animal would immediately initiate palpation of the screen upon hearing the white noise.

Once an animal learned the basic task, it was fitted with a removable blindfold (see below). Training continued $5 \mathrm{~d} /$ week until the animal routinely explored the screen for the wand, which was presented to the animal after progressively longer times after the start of the trial. Once the probe was introduced through the screen, animals easily located it within a few seconds. Eventually, animals would explore the screen continuously for $20-30 \mathrm{sec}$, performing $\sim 30$ such trials during a single training session. This typically required 3-4 weeks.

\section{Surgical preparation}

Rats underwent two surgeries. In the first, a dental acrylic appliance was attached to the skull for holding an amplifier base, microdrive, and blindfold. Two to 3 weeks later, a second surgery was performed to insert EMG electrodes in the mystacial pad and to position the electrode assembly over the appropriate part of the brain. Anesthesia was induced with metofane followed by halothane at $1-2 \%$. All surgical procedures were performed under sterile conditions.

During the first surgery, a skin incision was made over the skull, the underlying connective tissue was cleared, and the skull was thoroughly cleaned with $100 \% \mathrm{H}_{2} \mathrm{O}_{2}$. Small stainless steel screws, one of which was attached to a ground wire, were inserted into the bone to serve as an anchor for an acrylic base. In addition to the amplifier connector, which was placed over the occipital bone, one or two stainless steel threaded posts were placed as far anteriorly as possible. These were used to hold a removable custom-fitted plastic (Orthoplast) blindfold. After surgery, the animal was administered a subcutaneous injection of Torbugesic (2 $\mathrm{mg} / \mathrm{kg}$; Fort Dodge Laboratories Inc., Fort Dodge, IA), and crushed baby aspirin was added to the water bottle in its home cage. An oral antibiotic was administered for 7-10 d after surgery.

EMG electrode placement. During the second surgery, a pair of Tefloncoated stainless steel wires ( 0.0045 inches) was inserted through a small skin incision in the cheek and tunneled subcutaneously to the deep intrinsic muscles of the mystacial pad (Carvell et al., 1991). Appropriate placement of the wires was determined by the ability to elicit small movements of one to three neighboring whiskers using low-threshold stimulus currents. The proximal ends of the electrode wires were tunneled under the skin to the back of the head and soldered to the amplifier connector on the skull. The skin incision on the cheek was sutured using 4-0 silk, and wound margins were treated with bacitracin ointment.
Microdrive placement. While still anesthetized, the animal was placed in a stereotaxic frame with non-traumatic ear bars. A small craniectomy, leaving the dura intact, was made over the ventrobasal thalamus or the cortical barrel field; both sites were approached from the skull dorsum. Receptive field-mapping procedures were used to locate the representation of rostral (i.e., arcs 3-5) whiskers in one of the midventral rows (C or D) (see Fig. 2 for whisker nomenclature), because these whiskers were most likely to contact the screen during the whisking task and because they were surrounded by other whiskers (e.g., rows $\mathrm{C}$ and $\mathrm{E}$ for a row $\mathrm{D}$ recording site). A miniature microdrive assembly was positioned over the appropriate area (for review, see Carvell et al., 1996). For cortical recordings, the microdrive was positioned so as to target neurons in middle cortical depths, and the electrode tip was retracted to lie just outside the dura. For thalamic recordings, the electrode was advanced into ventral posteromedial nucleus during initial mapping and then withdrawn $\sim 1 \mathrm{~mm}$ dorsal to the nucleus. A thin layer of bacitracin ointment was applied to the craniectomy site and covered with a thin layer of sterile bone wax. A protective shield made of lightweight plastic was placed over the apparatus. The animal was treated after surgery as above. In four animals, two experiments were conducted several weeks apart, one in each hemisphere.

\section{Recording and data collection}

Data collection occurred after the animal was fully recovered and was again performing the behavioral task, usually $2-5 \mathrm{~d}$ after the second surgery. EMG and unit recordings were obtained through dual-channel differential amplifiers that were incorporated into a head stage that plugged into the amplifier base. Conventional secondary amplification and filtering stages were used. The microelectrode was again advanced into the whisker representation while aurally monitoring responses evoked by manual whisker stimulation. Once the representation of an appropriate whisker was located, the position of the electrode tip was adjusted carefully over a period of several minutes to isolate a small number of units responding crisply to stimulation of the whisker. Considerable care was taken to identify the whisker evoking the strongest response, which is hereafter denoted as the principal whisker (PW). This delineation was always confirmed by at least two observers before the beginning of the recording experiment. From this point in the experiment, no adjustments were made to the position of the electrode.

EMG and unit records were stored on the two audio channels of the video recorder along with the video images of the behavior. Data were collected for 10-15 trials during which all of the whiskers on the face were intact. Subsequently, selected whiskers were trimmed to within 1-2 $\mathrm{mm}$ of the skin surface. The animal was gently restrained in an experimenter's hands, the PW was reconfirmed, and whiskers were carefully trimmed using blunt surgical scissors. In all but three experiments, the eight whiskers surrounding the PW were trimmed (Fig. 1); the other cases involved trimming the PW alone or, inadvertently, in combination with some adjacent whiskers (see Results). After a 10-15 min rest, the rat performed an additional 10-15 trials.

At the conclusion of the experiment, an electrolytic lesion $(7 \mu \mathrm{A}$ for 7 sec) was made to mark the recording site (Fig. 1). Forty-five to $60 \mathrm{~min}$ later, the animal was deeply anesthetized with pentobarbital sodium, and the electrode was carefully withdrawn. The rat was then perfused for cytochrome oxidase histochemistry (Land and Simons, 1985a). Subsequently, the brain was frozen and cut in $60 \mu \mathrm{m}$ sections. For cortical experiments, the brain was sectioned tangential to the pial surface overlying the barrel field. For two of three thalamic experiments, the thalamus was sectioned in a standard coronal plane. In one case, the diencephalon was oriented in an oblique horizontal plane during sectioning so that individual thalamic barreloids could be visualized (Land and Simons, 1985b).

\section{Data analysis}

To identify periods of time when the rat's face was in immediate proximity to the screen and vigorously exploring it for at least $10 \mathrm{sec}$, each videotape was viewed at normal speed and on a frame-by-frame basis. This was done while listening to the simultaneous audio recordings of the neural records to verify that the spike activity correlated with screen exploration and that it clearly decreased when the animal moved away from the screen. Spikes were carefully inspected using a digital oscilloscope and amplitude discriminator to obtain an estimate of the number and shapes of isolatable units. Multi-unit and EMG records were then digitized at $50 \mathrm{kHz}$ and $500 \mathrm{~Hz}$, respectively, using DataWave software (Data Wave Technologies Corp., Longmont, CO). Unit wave- 

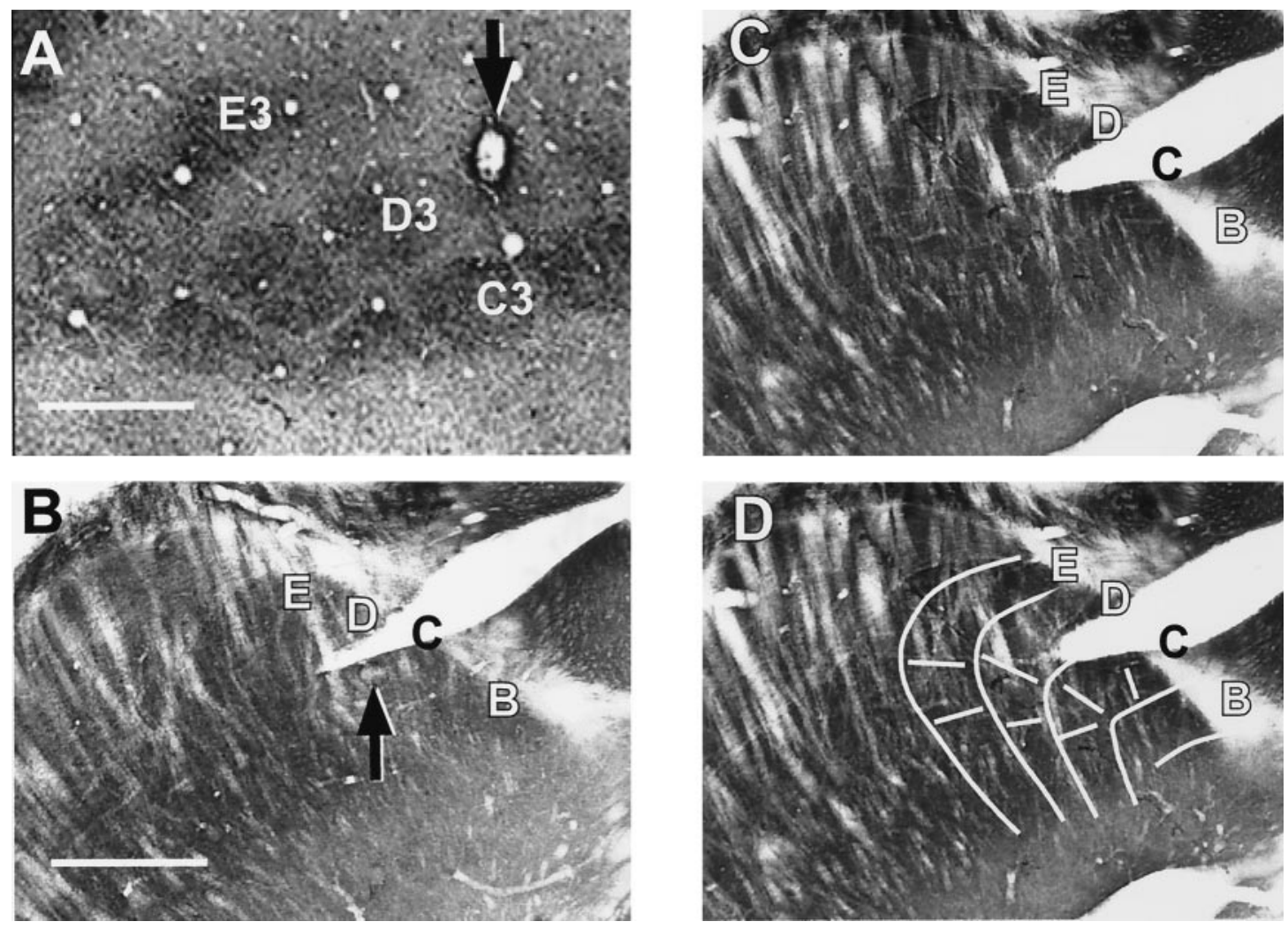

Figure 1. Recording site identification. $A$ shows cortical barrels in a $60 \mu \mathrm{m}$ tangential section from an experiment in which the PW was identified as D4. An electrolytic lesion made at the recording site (arrow) is located just superficial to the cytochrome oxidase-rich center of the D4 barrel. Top, Anteromedial. $B-D$ show histology from a thalamic recording experiment in which the PW was identified physiologically as $C 3$ (see Fig. 7). $B$ and $C$ show consecutive $60 \mu \mathrm{m}$ cytochrome oxidase-stained sections cut in an oblique horizontal plane (from dorsomedial to ventrolateral), a plane in which thalamic barreloids can be visualized (Land and Simons, 1985b, their Fig. 1C). Top, Dorsolateral; right, posterior. In B, an electrolytic lesion is visible in the C3 barreloid (arrow), and the electrode track is visible in C2 (immediately below the letter $C$ ). The arrangement of barreloid rows and arcs are more evident in $C$, which is the superficially adjacent section. $D$ shows the same section as in $C$ overlaid with a line drawing illustrating the overall orientation of barreloid rows B-E; row A can only be observed in deeper sections. The split in the tissue, partially transecting the row C barreloids, is an artifact of tissue processing, not an electrode track. Scale bars, $500 \mu \mathrm{m}$.

forms, which were always initially negative, were parsed from the continuous record by acquiring 32 points of the waveform centered around an amplitude threshold crossing of the negative-going phase. Thresholds were set conservatively to acquire data from typically the one to three largest amplitude units. Further analyses were performed using custom software.

Spike sorting. To extract single-unit data from the multi-unit record, we modified the procedure described by Fee et al. (1996). Parsed waveforms were examined visually to determine which segment of the 32 sample points contained the most stable region of the action potentials and at what time point in the sample the peak amplitude should be set to best capture the action potential waveforms. Unused sample points at the beginning and end of the original parsed record were discarded. Using cubic spline interpolation, the true peak of the action potential was estimated, and the waveform was resampled to 32 points so as to place the peak at a chosen sample time in the interpolated waveform. This procedure eliminates jitter in the aligning of waveforms that have been digitally sampled.

Spike waveforms were sorted using a variant of the method described by Fee et al. (1996) for stereotrode recordings (64 sample points across two electrodes). For the single-electrode data used in the present study, spike waveforms were treated as 32 element vectors. A subset $(\sim 4000)$ of all of the spike waveforms (i.e., spikes taken from one behavioral trial) were initially sorted into a large number of clusters (usually 16) by recursive bisection. For the first bisection, a small amount of gaussian noise was added to an average of all the waveforms to produce a second mean waveform. Each waveform was subsequently assigned to the one (of two) mean waveform that was closest to it in shape, updating the mean as each waveform was added. The assignment and updating procedure was repeated three times to allow the means to attain an equilibrium. Other bisections proceeded similarly. The resulting clusters were then combined into final single-unit clusters based on similarity of waveform shape and on a minimal interspike interval of $2.0 \mathrm{msec}$, corresponding to the refractory period. The final clusters were then used to form spike waveform templates to which we attempted to assign all parsed waveforms in the full data set. Waveforms that could not be assigned to one of the templates were discarded or, occasionally, resorted to identify infrequently spiking units.

In the method of Fee et al. (1996), spike waveforms are first sorted into initial clusters solely on the distance between the waveform vector and the cluster mean; the interspike interval criterion is applied only at the final stage of cluster aggregation. In our implementation of the method, after the first bisection and before each subsequent one, all spikes in the data subset were reassigned to the initial clusters on the basis of both waveform and timing criteria; a spike could be assigned to a cluster only if its waveform fell within a set distance from the cluster mean (based on a user selectable criterion ranging from 1.5 to $2.5 \mathrm{SDs}$ ) and only if the spike occurred at least $2.0 \mathrm{msec}$ later than any previously assigned spike. Similarly, both variance-based limits and interspike interval criteria were used in the final aggregation of the initial clusters and in the subsequent assignment of spikes to the final templates.

Data reduction. Spike occurrence times (saved at $100 \mu \mathrm{sec}$ resolution) and EMG data were analyzed in two ways. In the first, each electrophysiological record, corresponding to individual behavioral trials, was divided into sequential $100 \mathrm{msec}$ epochs. For each epoch, we summed the total number of spikes and computed the root-mean-square value of the EMG (Basmajian and DeLuca, 1985). The second analysis used an algorithm for detecting EMG bursts to distinguish periods of active whisking from periods in which whisking was of low amplitude or absent altogether. EMG signals were rectified and then smoothed by computing, 
for each sample point, a mean based on the sample point and the four points before and after it $( \pm 8 \mathrm{msec})$. The mean and variance of the rectified, smoothed EMG was computed for the entire trial. These values were used to establish thresholds for determining epochs during which the amplitude of the EMG signal remained within a chosen range of values (typically between 0.5 and 5.0 SDs above the mean). Successive EMG sample points occurring within this range were summed, and, after examining the distribution of these sums and the corresponding EMG traces, a criterion was chosen reflecting a minimal acceptable amount of integrated within-burst activity. In addition to the amplitude and size criteria, a valid burst could not begin within $25 \mathrm{msec}$ of the end of a previous one.

Spike counts were made for a $200 \mathrm{msec}$ period bracketing the EMG burst, beginning $50 \mathrm{msec}$ before the EMG signal crossing the lower amplitude threshold. Rectified, integrated EMG activity was summed over the same time periods. Note that, because whisking occurs at frequencies of 7-10 Hz, overlap can exist between the end of one such epoch and the beginning of the next. A "periburst time histogram" was constructed across all bursts and trials by accumulating spikes in $2 \mathrm{msec}$ bins, and averages were made of the EMG signals. Periods without whisking were identified as follows. The EMG record was sequentially examined to identify $200 \mathrm{msec}$ epochs during which no valid EMG burst occurred, and this was repeated every $50 \mathrm{msec}$ along the record until a burst was encountered. The search resumed at a randomly selected data point $50-100 \mathrm{msec}$ after the end of a burst. The variable starting time was used to avoid introducing periodicities into the non-burst associated spike and/or EMG data attributable to preceding periods of rhythmic bursting. Because of the variability in EMG signal amplification, which we optimized but did not calibrate for each recording session, all EMG data will be presented in arbitrary units of magnitude.

Data were analyzed to compare pre-trim and post-trim spike count and EMG measures. For each animal, this typically involved mean data obtained from several thousand time epochs. Within-animal comparisons were made using $t$ tests. Groups of animals were categorized according to recording location (thalamus and cortex) and whisker trimming [PW and adjacent whiskers (ADJ)]. Within-group comparisons of pre-trim and post-trim data were made using paired $t$ tests. Between-group comparisons were performed as follows. For each unit, we calculated the proportional change in unit activity as the ratio of post-trim to pre-trim values (hereafter denoted as Post/Pre ratios) and used $t$ tests to compare the mean values between groups. For all statistical tests, $p \leq 0.05$ (two-tailed) was used as the criterion for significance. Error measures are presented as SDs.

\section{RESULTS}

\section{General observations}

Date were obtained from 21 experiments in 17 animals. Recordings yielded 48 single units, which were studied under the following conditions of recording location and whisker trimming: cortex-PW trim ( $n=9$ units in 4 experiments); cortex-ADJ trim ( $n=33$ in 14 experiments); and thalamus-ADJ trim ( $n=6$ units in 3 experiments). EMG recordings were obtained for all but one (thalamic) experiment. Results are based on the analysis of 14,410 and 16,568 sec of pre-trim and post-trim data, respectively. Of the 42 cortical units, 20 and 17 were determined histologically to have been recorded in layers III and IV, respectively. Two recording sites (one unit each) were in the infragranular layers. Three units from one experiment could not be definitively located because of poor histology. None of the data analyses indicated laminar-dependent differences in the obtained results, and therefore data are pooled across layers for statistical analyses. Differences in receptive field properties across cortical layers might be revealed in a larger sample of units distributed more equally throughout the cortical depths.

Figure 2 illustrates the basic experimental paradigm and one of the main findings. In this animal, units were recorded from the C4 cortical column during eight trials when all whiskers were intact. Then, all of the immediately adjacent whiskers were trimmed to within a few millimeters of the skin surface, and the animal
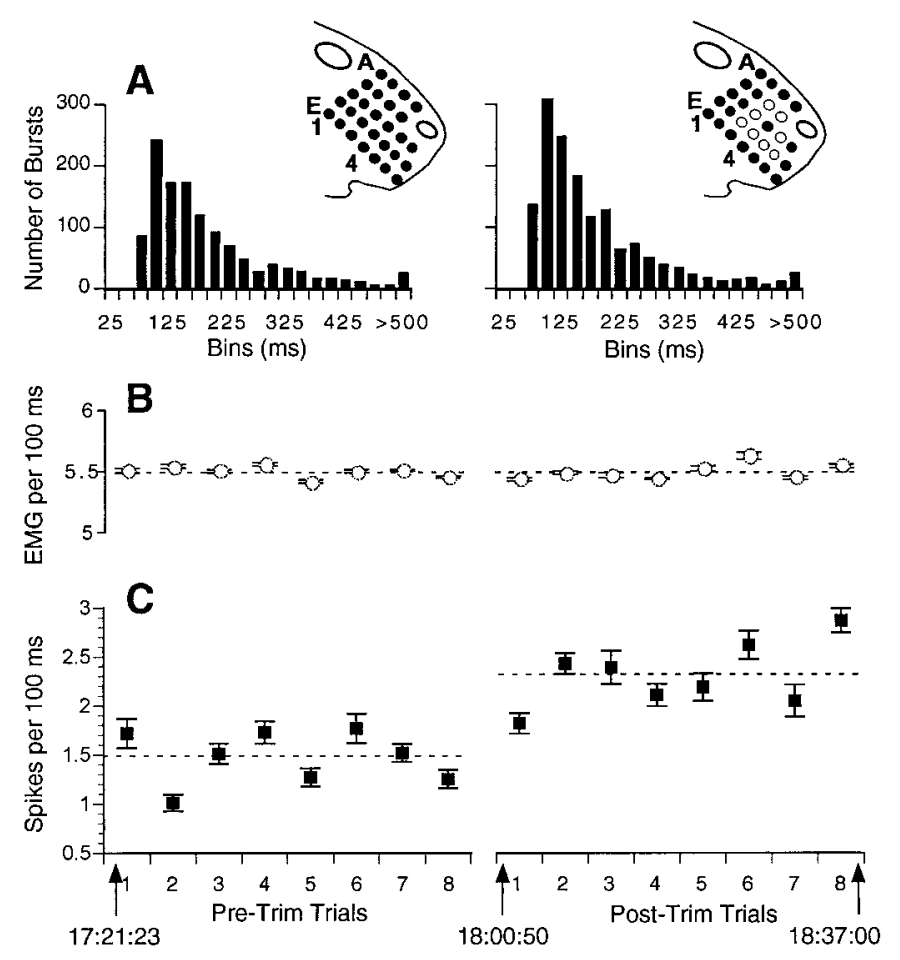

Figure 2. Schematic of experimental paradigm showing spike counts and EMG activity during pre-trim and post-trim trials. The figurines of the face illustrate whisker nomenclature. In the right figurine, the central solid circle indicates $\mathrm{C} 4$, the $\mathrm{PW}$, and the open circles indicate the immediately adjacent whiskers, which were trimmed before obtaining post-trim data. Graphs show mean \pm SE spike counts $(C)$ and integrated EMG $(B)$ averaged over all consecutive $100 \mathrm{msec}$ epochs during each trial. In this experiment, the animal performed eight pre-trim and eight post-trim trials. Note that the mean unit activity (indicated by the dashed line) increased after removing the whiskers surrounding the PW, but the overall EMG activity remained unchanged. Time stamps show the time course of the experiment. Histograms in $A$ show the distribution of intervals between successive EMG bursts.

performed an additional eight trials. Graphs show, on a trial-bytrial basis, the mean spike count per $100 \mathrm{msec}$ epoch of one unit (Fig. $2 C$ ) and two measures of the muscle activity recorded in the mystacial pad $(A, B)$. After trimming, the unit activity increased. Trial-by-trial comparisons indicate that spike counts are correlated with relative levels of EMG. This suggests that, in general, as whisking increases, there is more stimulus-evoked activity and that our methods are sensitive enough to detect it. Average EMG values $(B)$ during pre-trim and post-trim trials did not differ, indicating that the increased unit activity cannot be accounted for by changes in the overall level of whisker muscle activity. There was no difference in the distribution of interburst intervals for pre-trim and post-trim trials $(A)$, and in both cases the modal value was $100 \mathrm{msec}$.

Figure 3 illustrates the nature of the raw data. The bottom two traces show cortical unit and electromyographic activity obtained during a $1 \mathrm{sec}$ period when an animal was actively whisking the screen. Comparison of the two traces reveals an overall correspondence between the occurrence of EMG bursts and spike discharge (Fig. 4A). The expanded traces at the top show individual action potentials. The spike-sorting program distinguished three units from this experiment, representative waveforms of which are shown. Synchronized EMG and spike data from one of these units (Fig. 3, asterisks) is shown in Figure $4 A$. The left panel 


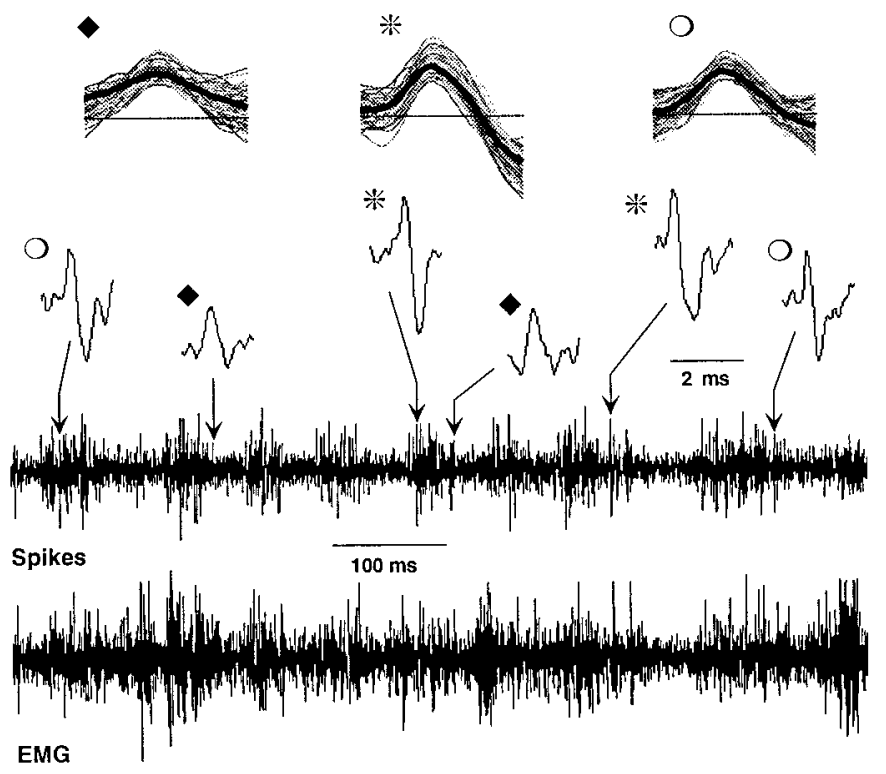

Figure 3. Representative raw data. The bottom two traces show EMG and neural traces during a $1 \mathrm{sec}$ period of whisking. Note overall correspondence of spike discharges with EMG bursts. Three units were isolated from the neural records; two occurrences of each are shown in the expanded view above the continuous neural record (note $2 \mathrm{msec}$ scale). Top traces show 100 consecutive waveforms from each unit along with the templates (thick dark lines) to which the waveforms were assigned. Units were recorded at the site of the lesion in the D4 barrel shown in Figure $1 \mathrm{~A}$.

shows average EMG burst traces and corresponding periburst time histograms, and the right panel shows activity obtained during periods without EMG bursting. A clear correspondence between EMG bursts and unit discharge is apparent.

In this experiment, the PW was identified as the D4 whisker on the basis of manual stimulation applied while the rat was awake and gently restrained by hand. Subsequent histological reconstruction confirmed that recordings were obtained from the D4 column at the layer III/IV border (Fig. 1A). After obtaining baseline data (pre-trim), the PW was trimmed. As illustrated in Figure $4 A$, unit activity was profoundly diminished both during periods of active whisking (measured as EMG burst) and during screen contact without whisking (measured as the absence of EMG bursts). The average EMG traces are virtually indistinguishable during pre-trim and post-trim trials, suggesting that changes in the behavior cannot readily account for the pronounced decrease in unit activity.

Representative data from the other two experimental conditions are shown in Figure 4, $B$ and $C$. Results in $B$ were obtained from a cortical recording during which the eight whiskers surrounding the PW were trimmed. In contrast to removal of the PW, removal of adjacent whiskers led to a $45 \%$ increase in unit activity during both whisking and non-whisking epochs. In this animal, average EMG values were actually lower during the post-trim trials. Opposite effects of adjacent whisker removal are observed in the thalamic unit, whose results are illustrated in $C$. Post-trim neural activity was $77 \%$ lower than pre-trim activity, despite virtually no difference in overall EMG.

An interesting aspect of the data in the left panels of Figure 4 is the temporal relationship between (pre-trim) unit activity and EMG burst. Both cortical units displayed temporally focused activity peaks that occurred near maximal whisker protraction (as inferred from the EMG record; Carvell et al., 1991). Based on the

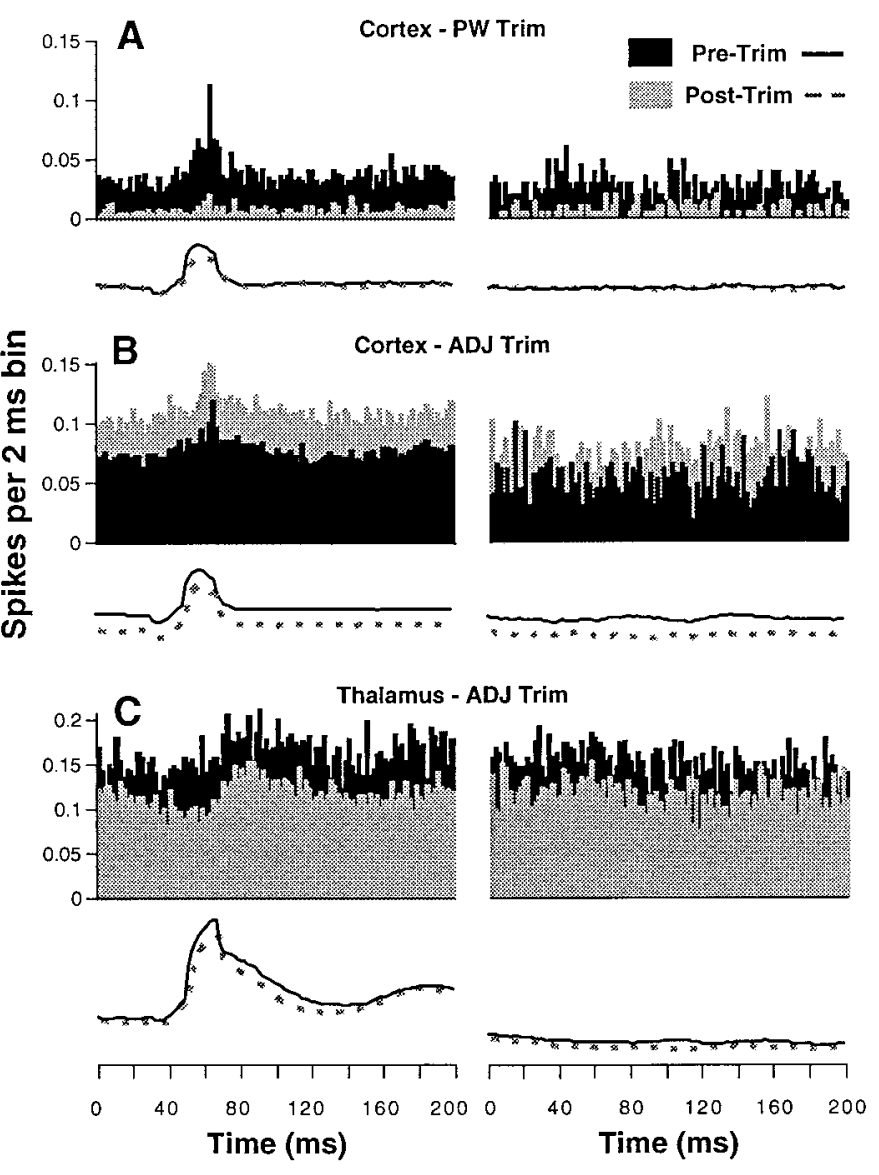

Figure 4. Periburst time histograms. Each panel shows averaged EMG activity (traces) and corresponding unit activity accumulated in time histograms having $2 \mathrm{msec}$ bins. Data on the left are taken from $200 \mathrm{msec}$ epochs containing EMG bursts and are aligned with respect to burst onset, which occurs at the 50th msec bin. EMG averages (scales in arbitrary units not shown) were made from rectified signals, and bursts were identified using threshold and magnitude criteria (see Materials and Methods). Data on the right were obtained during periods of screen contact without EMG bursting. Pre-trim (black) and post-trim (light gray) data are shown for representative units under the three experimental conditions. Note the opposite effects on cortical and thalamic unit activity with ADJ trimming.

video and audio records, these peaks correspond to contact of the whiskers with the screen. The response profile for the thalamic unit (Fig. 4C) differs in two respects; relative to the peak of the EMG burst, unit activity occurs later, and it is more temporally dispersed. Such temporally offset peaks were not unique to thalamic units, because they were observed also in some cortical recordings. Differences in peak response time may be related to the directional sensitivity of the individual units, but we were unable to assess this accurately in the loosely restrained animal. Cortical units also varied with respect to the temporal dispersion of their response peaks. Approximately $60 \%$ displayed profiles similar to those in Figure $4, A$ and $B$, with the remaining unit profiles being weakly modulated or completely unmodulated by presumed whisker contact. Even in the latter cases, however, the total neural activity at the chosen recording sites was always clearly related to manual whisker deflections. In contrast, the smaller sample of thalamic units were less strongly modulated. In fact, the data shown in Figure $4 C$ represent the most strongly modulated profile of the four thalamic units for which EMG records were obtained. 

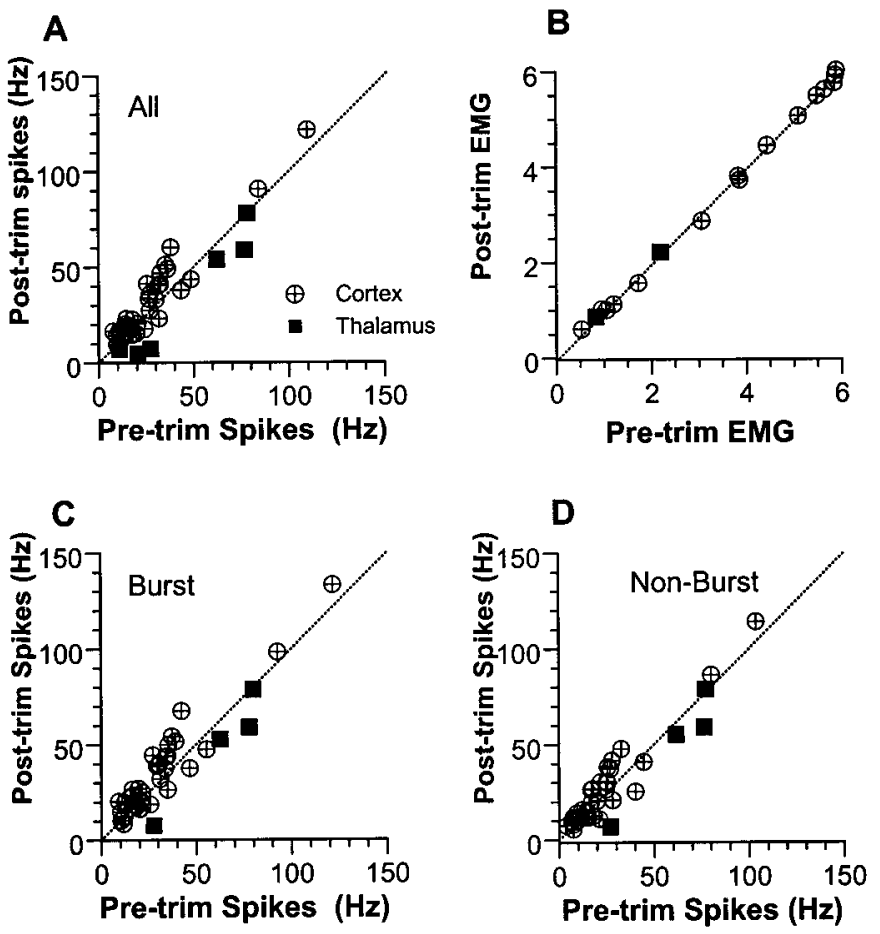

Figure 5. Scatterplots showing effects of adjacent whisker trimming on cortical and thalamic units. Average spike $(A)$ and EMG $(B)$ activities measured in consecutive $100 \mathrm{msec}$ epochs. Spike counts are converted to frequency; EMG is measured in arbitrary units. Spike activities measured during 200 msec burst $(C)$ and non-burst $(D)$ epochs (see Fig. 4, left and right). Dotted lines represent a 1:1 correspondence. Note that, overall, spike activities are highest in $C$ (burst), lowest in $D$ (non-burst), and intermediate in $A$ (undifferentiated).

\section{Quantitative analyses}

The principal issue addressed in the present experiments concerns the immediate effects on a barrel-related column of removing adjacent whiskers while leaving the principal whisker intact. Quantitative data for the cortical and thalamic neurons studied using this experimental manipulation are presented in Figure 5. $A$ presents findings based on average spike counts obtained in all $100 \mathrm{msec}$ epochs during pre-trim and post-trim trials. With all whiskers intact, cortical units discharged at a mean rate of $27.6 \pm$ $21.0 \mathrm{~Hz}$, which increased to $32.1 \pm 23.8 \mathrm{~Hz}$ after adjacent whisker removal ( $p=0.001 ; n=33$ ). Thalamic units fired at an average of $46.0 \pm 29.9 \mathrm{~Hz}$ with all whiskers present, and decreased their firing after whisker trimming to $35.0 \pm 32.4 \mathrm{~Hz}(p=0.02 ; n=6)$. $B$ presents average root-mean-square values for EMG activity in the same $100 \mathrm{msec}$ bins; the number of observations is smaller than in $A$ because the same EMG data were used for all units in a given experiment. Mean pre-trim and post-trim EMG values (in arbitrary units) are highly similar for both cortical (3.64 $\pm 2.05 \mathrm{vs}$ $3.61 \pm 2.06 ; n=14)$ and thalamic experiments $(1.51 \pm 0.96 \mathrm{vs}$ $1.54+0.96 ; n=2)$.

For the preceding analyses, data were simply compiled from consecutive $100 \mathrm{msec}$ epochs without regard to the presence or absence of whisking, as defined by EMG bursts. As shown in Figures 2 and 4, overall neural activity is higher during periods of EMG bursting. To rule out the possibility that observed changes in neural activity reflect the relative incidence of EMG bursts (whisks) during pre-trim versus post-trim trials, we parsed the data records into burst and non-burst epochs (see Materials and
Methods). Figure 5C shows data obtained during periods of EMG bursts; values represent average firing rates computed over a 200 msec epoch bracketing the burst. After adjacent whisker removal, cortical units increased their activities during both burst (30.4 \pm 23.2 vs $35.4 \pm 25.6 ; p=0.001 ; n=33$ ) and non-burst epochs $(23.1 \pm 20.4$ vs $25.9 \pm 22.5 ; p=0.025)$. In the thalamus, EMG records were available for four units only. On average, their activities decreased but not significantly (burst, $62.0 \pm 24.2$ vs $49.5 \pm 30.0 ; p=0.063$; non-burst, $60.6 \pm 23.5$ vs $50.0 \pm 30.5 ; p=$ $0.124)$. As for the $100 \mathrm{msec}$ bin analyses, there were no statistically significant differences between pre-trim and post-trim EMG activity for either cortical or thalamic experiments.

Data from cortical and thalamic units were compared directly using mean ratios of post-trim to pre-trim activity. For each measure, the two groups of units differed significantly, with cortical units displaying mean ratios $>1.0$, indicative of increased activity, and thalamic units displaying mean ratios $<1.0$. For the $100 \mathrm{msec}$ bin data, the average increase in cortical unit activity was $20 \%$ compared with a $37 \%$ decrease in thalamic activity $(1.20 \pm 0.33$ vs $0.63 \pm 0.31 ; p<0.001 ; n=33$ and 6$)$. Virtually identical values were obtained for cortical activity measured during 200 msec burst $(1.21 \pm 0.34)$ and non-burst $(1.17 \pm 0.33)$ epochs. For both measures, these values differed significantly from the smaller set of four thalamic units for which EMG data were available (burst, $0.72 \pm 0.31 ; p=0.008$; non-burst, $0.74 \pm$ $0.34 ; p=0.016)$.

For each animal, we computed the mean interval between EMG bursts. Values were similar for pre-trim and post-trim trials $(248 \pm 71$ vs $242 \pm 72 \mathrm{msec} ; p=.28)$, and Post/Pre ratios did not differ between thalamic and cortical experiments $(0.89 \pm .005$ vs $0.99 \pm 0.18 ; p=0.55)$. The largest change was observed in a thalamic experiment in which the mean interburst interval decreased from 337 to $245 \mathrm{msec}$; despite this increase in overall whisking frequency, two of the three units recorded at this site decreased their activities by 13 and $23 \%$, with the other unit displaying no change. Modal intervals, determined using frequency histograms having $25 \mathrm{msec}$ bins, averaged $112.5 \mathrm{msec}$ for both pre-trim and post-trim trials $(p=1.0)$. For any given experiment, the modal interburst interval did not vary by more than one bin before and after whisker trimming.

Data were also analyzed separately for each unit. Two-tailed Student's $t$ tests were used to determine whether differences existed between pre-trim and post-trim spike counts. Data compiled from consecutive $100 \mathrm{msec}$ epochs are presented in Figure 6 . Of the 33 cortical units examined after adjacent whisker removal, 64\% displayed significant increases in unit activity (mean of $38 \%$ ), 24\% showed a significant decrease (mean of 19\%), and $12 \%$ were statistically unchanged. Interestingly, at one recording site, one of the two isolated units significantly increased its firing, whereas the other significantly decreased its firing. In contrast, none of the thalamic units studied under these conditions increased its firing. Of the six thalamic units, five (83\%) significantly decreased their activity (mean of $45 \%$ ), the remaining unit showing no change. Units were categorized as having increased activity or not (activity decrease or no change). A $\chi^{2}$ test comparing the frequency of these categories revealed a significant difference between thalamic and cortical units $(p<0.001)$. Removal of the PW resulted in a decrease in cortical unit firing for eight of nine units (mean of 59\%). One unit showed a statistically significant increase of $20 \%$; this unit was recorded at a site in which a second unit showed a significant activity decrease. 

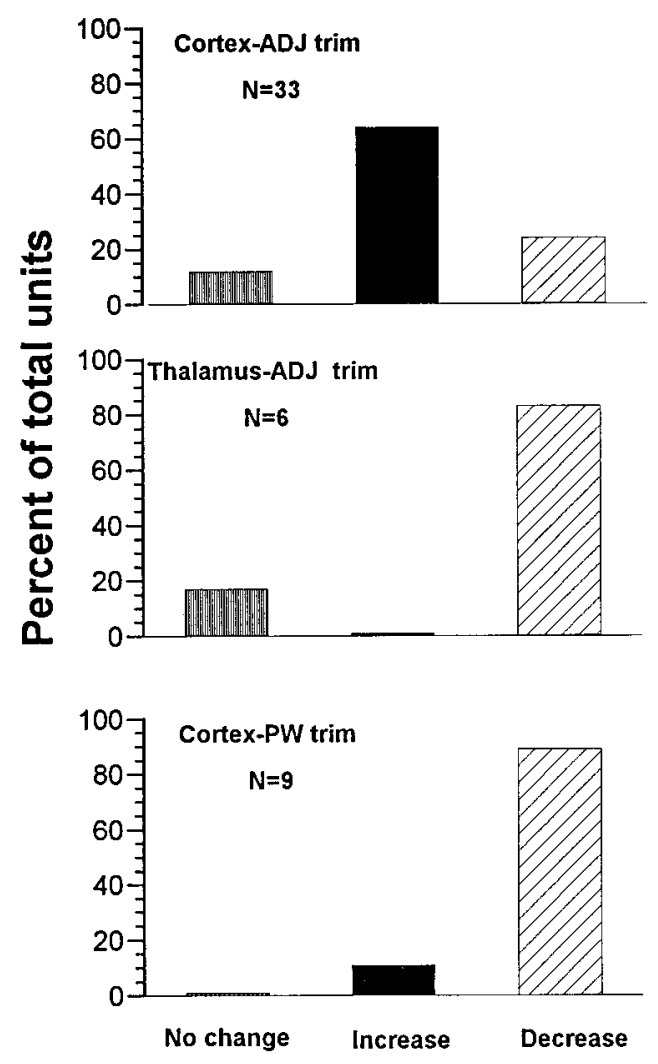

Figure 6. Summary of statistical comparisons performed on individual units. Student's $t$ tests were used to compare pre-trim and post-trim spike activity (100 msec epochs) for all units in the three experimental conditions. Shown are percentages of each population showing statistically significant changes $(p<0.05$; two-tailed).

\section{Determining the principal whisker in awake animals}

Interpretation of the present results critically depends on identification of PW versus adjacent whiskers in the awake animal, using manual stimulation and audio monitoring. For virtually all cortical recording sites, the PW was readily distinguished from its neighbors by the clearly more vigorous responses its deflection evoked in the overall neural activity. Histological reconstruction of the cortical barrel field provided confirmation that the physiologically identified PW corresponded with the anatomical representation of the whisker. The one exception in the cortical data was a recording site in which physiological identification of the PW was ambiguous, because the units responded equally well to a number of neighboring whiskers, one of which we selected as the PW based on the slightly stronger response it evoked. This site was subsequently found to be within the interbarrel septum close to the barrel corresponding to the physiologically identified PW. We therefore operationally classified this experiment as a "PW trim." Removal of its neighboring whiskers resulted in a 56\% decrease in unit activity, consistent with the strong multi-whisker nature of septal receptive fields.

In the thalamic recording experiments, physiological identification of the PW was considerably more difficult, because the receptive fields of the overall neural activity were consistently multi-whisker. In the final experiment, we confirmed the physiological identification of the PW after the recording session using controlled whisker deflections while the rat was anesthetized with halothane. In this experiment, we also sectioned the thalamus in an oblique horizontal plane to identify the barreloid in which the
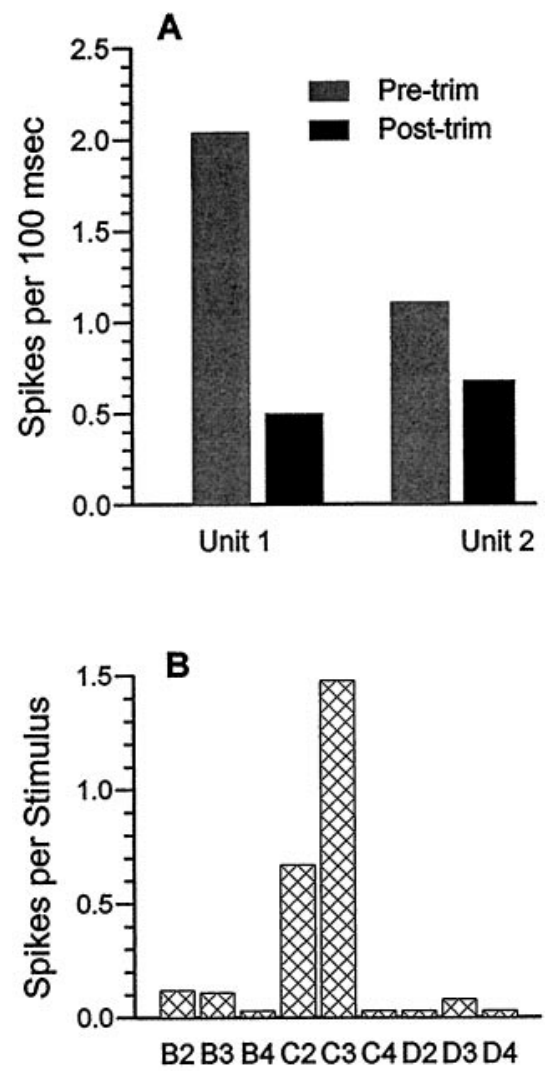

Figure 7. Data from an identified thalamic barreloid. $A$, Effects of adjacent whisker trimming on two thalamic units recorded at the lesion site in the $\mathrm{C} 3$ barreloid shown in Figure $1 B$. Note decreased activity in both cases after trimming. $B$, Confirmation of $\mathrm{C} 3$ as the PW. After behavioral testing, the rat was anesthetized with halothane, and controlled whisker stimuli (Simons, 1983) were applied individually to C3 and each of the eight surrounding whiskers. Spike counts were obtained from the multi-unit record with the amplitude threshold set high to discriminate the two largest units, which corresponded to the individual units of $A$. The C3 whisker was trimmed to the same length as the previously clipped adjacent whiskers.

recordings were obtained. In the awake condition, neural activity was evoked by several whiskers in the vicinity of C3, with the C3 and $\mathrm{C} 2$ whiskers evoking the most vigorous responses. After careful testing, we designated $\mathrm{C} 3$ as the PW, subsequently trimming the eight whiskers surrounding it. After the animal was anesthetized, the receptive field at this recording site became clearly focused on the $\mathrm{C} 3$ and $\mathrm{C} 2$ whiskers (Fig. 7B).

Figure $1, B-D$, shows histology from this animal. The marker lesion was appropriately located in the C3 barreloid. The spikesorting program identified two discriminable units. Results of adjacent whisker removal on their activities during the behavioral task are presented in Figure $7 A$. The EMG wires had apparently become displaced since their insertion several days previously, so that EMG data are not available from this animal. For both units, spike counts per $100 \mathrm{msec}$ epochs decreased significantly after adjacent whisker removal.

\section{DISCUSSION}

A unique feature of the rodent somatosensory cortex is the anatomic correspondence between barrels and whiskers. Numerous electrophysiological studies using passive whisker stimulation in immobilized animals, with or without general anesthesia, have demonstrated a functional correspondence as well. Specifically, 
within a vertical column centered on a layer IV barrel, neurons at different cortical depths respond only or maximally to the whisker that is anatomically appropriate to that barrel (Simons and Woolsey, 1979; Armstrong-James and Fox, 1987). This correspondence between cortical anatomy and function has been demonstrated previously in naturally exploring animals using markers for metabolic activity (Durham and Woolsey, 1978; McCasland and Woolsey, 1988). In those studies, acute removal of selected whiskers led to reduced 2DG labeling in their corresponding barrels. Moreover, subtle changes in the labeling pattern across the barrel field paralleled adjacent whisker-related inhibitory gradients observed in neurophysiological studies (McCasland et al., 1991). The present findings extend these observations by demonstrating that, in the behaving rat, the spike activity of neurons within the anatomic map of the whiskers can be clearly ascribed to specific whiskers. As expected, removal of the principal whisker leads to a substantial decrease in unit activity in the corresponding barrel and its associated column. Less intuitively, this manipulation leads to an increase in unit activity in neighboring columns. We interpret this latter finding to reflect disinhibition.

\section{Methodological issues}

Our motivations for developing the screen exploration task were twofold. First, the task had to require the rat to use its whiskers vigorously, providing strong self-activation of the sensory system. Second, the task had to involve a behavior that was relatively natural, easy to perform, and minimally stressful. These considerations were important because activity from the same neurons were to be compared under conditions when all whiskers were intact and again after the immediate removal of as many as $25 \%$ of the large whiskers on the mystacial pad. Thus, failure of an animal to perform the task after trimming, perhaps as a result of stress caused by the absence of whiskers, would eliminate inclusion of its data from the analyses, despite a lengthy period of previous preparation. Moreover, whisker removal is for practical purposes the end point of the experiment, because several weeks are required for regrowth, a period during which electrode tracks and marker lesions might disappear.

Previous studies from our laboratory that investigated whisking behavior involved detailed kinematic reconstructions of video images, made on a frame-by-frame basis (Carvell and Simons, 1990). To visualize individual whiskers, these analyses required the animal to behave within a physically limited field of view while focusing their whiskers on a relatively challenging set of discriminanda. Because these types of analyses and behaviors were poorly suited to the present experimental goals, we opted for extensive, prolonged screen exploration rather than high-resolution whisker imaging. The explicit assumption is that data collection spanning multiple trials (each lasting tens of thousands of milliseconds) would permit a reliable estimate of unit activity that is relatively independent of moment-to-moment variations in the extent to which the whiskers contact the screen. In addition, we used several control measures to ensure that trimming-induced changes in unit activity did not simply reflect systematic changes in the behavior. First, the size of the screen was chosen to permit adequate video resolution for identifying periods during which the animal was exploring it, and data were analyzed only during these epochs. Second, we directly measured muscle activity from the mystacial pad contralateral to the unit recording location and analyzed the unit data in three related, but different, contexts: overall EMG activity; the presence of EMG bursts, indicative of active whisker protraction; and the presence of non-bursting low-level activity, corresponding to periods when whiskers were being passively drawn across the screen by head movement. Third, we recorded from another location in the whisker-tobarrel pathway, i.e., thalamic barreloids, and found the effects of adjacent whisker removal to be different from those in the cortex. Ideally, unit recordings should be obtained simultaneously from homologous barreloids and barrels in the same animal, but we were unsuccessful in several attempts to do this. Fourth, although the 100 msec spike count data of Figure 5 suggest that the same qualitative results of whisker trimming would have been observed if we had non-selectively incorporated the two or three largest amplitude spikes in the measured neural record, the use of a spike-sorting procedure provides additional evidence that the observed effects of whisker removal are caused by changes in neural responsiveness and not by changes in whisking behavior. At several recording sites, post-trimming activity was observed to be elevated for one unit but decreased for another.

\section{Comparison with previous characterizations of whisker-related receptive field organization}

Previous single-unit studies of fentanyl-sedated rats (Simons and Carvell, 1989; Brumberg et al., 1996) have revealed a number of important differences in the receptive field properties of thalamic barreloid and cortical barrel neurons. Relevant to the present findings, thalamic units, as a population, display multi-whisker excitatory receptive fields with weak adjacent whisker inhibitory effects. In contrast, the largest number of recorded barrel neurons (regular-spike units) are excited mainly or exclusively by the principal whisker, which also evokes the strongest inhibition within its parent barrel. Adjacent whiskers, deflected alone or in combination, also evoke "cross-whisker" inhibition, but unlike the PW, they evoke little or no excitation of regular-spike barrel units. Because of the strongly multi-whisker nature of their receptive fields, inhibitory ("fast-spike") units are thought to mediate crosswhisker inhibitory interactions locally, within their parent barrel (Kyriazi and Simons 1993; Pinto et al., 1996; Goldreich et al., 1999). Stimulus-evoked inhibition disproportionately diminishes excitatory responses evoked by weakly excitatory stimuli (Brumberg et al., 1996), as does direct microiontophoretic application of GABA itself (Kyriazi et al., 1996). For example, deflections of the PW can completely abolish the otherwise small excitatory responses associated with adjacent whisker stimulation. In light of these findings, Brumberg et al. (1996) proposed that, during active touch, simultaneous palpation of an object by multiple neighboring vibrissae would enhance signal contrast (e.g., "PWness") in barrel regular-spike neurons by disproportionately diminishing excitatory effects of non-columnar whiskers. Accordingly, when all whiskers are actively exploring an object, overall activity in a cortical barrel would be reduced relative to that evocable by deflections of whiskers singly. The activity that remains, however, would be more focused on the PW.

Here, we indirectly assessed the contribution of neighboring whiskers to cortical and thalamic activity by eliminating their influence. Consistent with the 2DG results of McCasland and Woolsey (1988) in freely exploring mice, acute removal of adjacent whiskers was associated with increased cortical unit activity in the barrel representing the intact PW. We interpret the results of these two studies as follows. In the absence of adjacent whiskers, the PW provides the nearly exclusive afferent excitatory drive to neurons in the homologous barreloid and barrel. Because the trimmed adjacent whiskers can no longer contact the screen, they are unable to contribute, along with the PW, to excitation in 
the thalamus and to "tonic" inhibition within the cortex. This yields PW-evoked responses that are less vigorous in the thalamus and more vigorous in the cortex, relative to when all whiskers are present. In the cortex, the disinhibitory effect is so pronounced that, in the present experiments, cortical neurons increased their activity an average of $20 \%$, despite an average $37 \%$ reduction in the firing of thalamic neurons.

\section{Implications for deprivation-induced plasticity}

A commonly used paradigm for studying plasticity in the somatosensory system involves the removal of whiskers by trimming, plucking, or follicle cauterization. When performed in normally reared adult animals, whisker removal has been associated with an increase in the activity evoked by the remaining whiskers (Diamond et al., 1993). In cases in which two adjacent whiskers are left intact, increased responses to both whiskers are observed within the same barrel after only a few days of deprivation. This phenomenon has been attributed to correlated neuronal activities as a result of whisker "pairing." The present findings indicate that the effect of selected whisker removal in an otherwise normally functioning animal is one of profound disinhibition of neurons in neighboring cortical columns, at least in the short term. Whether, and to what extent, such net disinhibition persists over time remains to be determined. Nevertheless, even short periods of disinhibition, lasting a few days, are likely to contribute substantially to alterations in receptive field properties. Indeed, reduction of tonic inhibition within local cortical circuits may be the primary causative factor in the cascade of events leading to synaptic modifications. For example, NMDA-dependent long-term potentiation requires membrane depolarization. Thus, changes in receptive field properties observed when all but two whiskers are chronically removed may be more a reflection of partial whiskerfield removal ("whisker sparing") than of whisker pairing. In neonatal animals, whisker removal results in a variety of physiological and behavioral abnormalities that persist despite longterm regrowth of the whiskers during adulthood (Simons and Land, 1987; Akhtar and Land, 1991; Carvell and Simons, 1996). These effects may reflect, at least in part, the absence of normal levels of inhibition during periods when sensory experience refines the cortical circuitry.

\section{REFERENCES}

Akhtar ND, Land PW (1991) Activity-dependent regulation of glutamic acid decarboxylase in the rat barrel cortex: effects of neonatal versus adult sensory deprivation. J Comp Neurol 307:200-213.

Armstrong-James M, Fox K (1987) Spatiotemporal convergence and divergence in the rat SI "barrel" cortex. J Comp Neurol 263:265-281.

Armstrong-James M, Fox K, Das-Gupta A (1992) Flow of excitation within rat barrel cortex on striking a single vibrissa. J Neurophysiol 68:1345-1358.

Basmajian JV, DeLuca CJ (1985) Muscles alive, their functions revealed by electromyography, p 97. Baltimore: Williams \& Wilkins.

Brumberg JC, Pinto D, Simons DJ (1996) Spatial gradients and inhibitory summation in the rat whisker barrel system. J Neurophysiol 76:130-140.

Buonomano DV, Merzenich MM (1998) Cortical plasticity: from synapses to maps. Annu Rev Neurosci 21:149-186.
Carvell GE, Simons DJ (1990) Biometric analyses of vibrissal tactile discrimination in the rat. J Neurosci 10:2638-2648.

Carvell GE, Simons DJ (1996) Abnormal tactile experience early in life disrupts active touch. J Neurosci 15:2750-2757.

Carvell GE, Simons DJ, Lichtenstein SH, Bryant P (1991) Electromyographic activity of mystacial pad musculature during whisking behavior in the rat. Somatosens Mot Res 8:159-164.

Carvell GE, Miller SA, Simons DJ (1996) The relationship of vibrissal motor cortex unit activity to whisking in the awake rat. Somatosens Mot Res 13:115-127.

Diamond ME, Armstrong-James M, Ebner FF (1993) Experiencedependent plasticity in adult barrel cortex. Proc Natl Acad Sci USA 90:2082-2086.

Durham D, Woolsey TA (1978) Acute whisker removal reduces neuronal activity in barrels of mouse SmI cortex. J Comp Neurol 178:629-644.

Fee MS, Mitra PP, Kleinfeld D (1996) Automatic sorting of multiple unit neuronal signals in the presence of anisotropic and non-Gaussian variability. J Neurosci Methods 69:175-188.

Fox K (1992) A critical period for experience-dependent synaptic plasticity in rat barrel cortex. J Neurosci 12:1826-1838.

Goldreich D, Kyriazi HT, Simons DJ (1999) Functional independence of layer IV barrels in rodent somatosensory cortex. J Neurophysiol 82:1311-1316.

Keller A, Carlson GC (1999) Neonatal whisker clipping alters intracortical, but not thalamocortical, projections in rat barrel cortex. J Comp Neurol 413:83-94.

Kyriazi HT, Simons DJ (1993) Thalamocortical response transformations in simulated whisker barrels. J Neurosci 13:1601-1615.

Kyriazi HT, Carvell GE, Brumberg JC, Simons DJ (1996) Quantitative effects of GABA and bicuculline methiodide on receptive field properties of neurons in real and simulated whisker barrels. J Neurophysiol 75:547-560.

Land PW, Simons DJ (1985a) Cytochrome oxidase staining in the rat SmI barrel cortex. J Comp Neurol 238:225-235.

Land PW, Simons DJ (1985b) Metabolic and structural correlates of the vibrissae representation in the thalamus of the adult rat. Neurosci Lett 60:319-324.

McCasland JS, Hibbard LS (1997) GABAergic neurons in barrel cortex show strong, whisker-dependent metabolic activation during normal behavior. J Neurosci 17:5509-5527.

McCasland JS, Woolsey TA (1988) High-resolution 2-deoxyglucose mapping of functional cortical columns in mouse barrel cortex. J Comp Neurol 278:555-569.

McCasland JS, Carvell GE, Simons DJ, Woolsey TA (1991) Functional asymmetries in the rodent barrel cortex. Somatosens Mot Res 8:111-116.

Micheva KD, Beaulieu C (1995) An anatomical substrate for experience-dependent plasticity of the rat barrel field cortex. Proc Natl Acad Sci USA 92:11834-11838.

Nicolelis MA, Lin RCS, Woodward DJ, Chapin JK (1993) Dynamic and distributed properties of many-neuron ensembles in the ventral posterior medial thalamus of awake rats. Proc Natl Acad Sci USA 90:2212-2216.

Pinto DJ, Brumberg JC, Simons DJ, Ermentrout GB (1996) A quantitative population model of whisker barrels: re-examining the WilsonCowan equations. J Comput Neurosci 3:247-264.

Simons DJ (1983) Multi-whisker stimulation and its effects on vibrissa units in rat SmI barrel cortex. Brain Res 276:178-182.

Simons DJ, Carvell GE (1989) Thalamocortical response transformation in the rat vibrissa/barrel system. J Neurophysiol 61:311-330.

Simons DJ, Land PW (1987) Early experience of tactile stimulation influences organization of somatic sensory cortex. Nature 326:694-697.

Simons DJ, Woolsey TA (1979) Functional organization in mouse barrel cortex. Brain Res 165:327-332. 